\title{
COMPARATIVE UTILITY OF BONE MARROW ASPIRATION AND BONE MARROW BIOPSY
}

Amrish Pandya, Tejas Patel, Neelam Shah

1. Professor \& Head, Department of IHBT, Government Medical College. Surat, Gujarat,

2. Senior Resident, Department of Pathology, Government Medical College. Surat, Gujarat,

3. Senior Resident, Department of Pathology, Government Medical College. Surat, Gujarat,

\section{CORRESPONDING AUTHOR}

Dr. Amrish Pandya, 702/b, Amrutdhara Apts, Opp. St. Xavier's School, Ghod Dod Road, Surat-1, Gujarat, India E-mail: dr_amrish_21@yahoo.com, Ph: 00919824196639.

ABSTRACT: The bone marrow examination is valuable in the diagnosis of certain hematological and non-hematological conditions and has been a cornerstone of hematology practice. Bone marrow can be obtained in two ways: 1.by needle aspiration and 2.by trephine biopsy.

Initially, aspiration techniques were more popular in view of the simplicity of the procedure, good representation of marrow cells' morphology and ready acceptability by the patient. However knowledge about diagnostic limitations of aspirated marrow, coupled with introduction of simplified percutaneous needle biopsy procedures (under local anesthesia) led to a progressive increase in the use of bone marrow trephine biopsy as an indispensable adjunct to bone marrow aspirations.

In this study we compared results and utility of bone marrow aspiration and bone marrow biopsy. Total 27 BMA and BMB were performed. BMA alone is sufficient in making diagnosis in $70 \%$ cases while remaining $30 \%$ cases required BMB for diagnosis. Bone marrow aspirate smears are ideal for the study of cytological details of haemopoietic cells. Trephine biopsies are superior to bone marrow aspirates particularly for the assessment of cellularity, extent and pattern of tumor infiltration and cell type. Valuable information on the status of hematopoiesis and changes in stromal components is also provided by trephine biopsy specimen.

Today, both procedures are considered complementary to each other.

KEY WORDS: BMA: bone marrow aspiration, BMB: bone marrow biopsy.

INTRODUCTION: Bone marrow examination is necessary investigation for diagnosis and management of many hematological and non hematological diseases. It is also useful in follow up of patient of chemotherapy and bone marrow transplants. For bone marrow examination two types of samples - BMA and BMB can be obtained. Marrow biopsy by surgical trephine is an older procedure than needle aspiration. It is only since the late 1950s that core needle biopsy of the bone marrow has been used [1] but BMA is simple, minimally invasive technique. However BMA has lower sensitivity in detecting solid tumour metastasis and lymphoma involvement in comparison to BMB. [2] BMB is more reliable method of detecting marrow infiltrate. ${ }^{\text {[3] And }}$ pattern of marrow involvement. For detecting cellularity of marrow BMB is more reliable than BMA. 
Nowadays aspirate and trephine biopsy specimens are considered complementary and when both are obtained, they provide a comprehensive study of bone marrow. ${ }^{[4]}$

MATERIALS AND METHODS:_The present study was carried out in the department of pathology, medical college. It comprises all the bone marrow biopsies performed on patients admitted to teaching hospital. Patients were reviewed irrespective of age and sex.

In each case a detailed history along with systemic, general and local examination and routine hematological investigation was carried out prior to bone marrow trephine biopsy.

The histopathology findings and the diagnosis made on the biopsies were compared with the findings on the bone marrow aspirate and diagnosis.

Patients were explained about the procedure. They were informed about possible complications but also assured for safety, simplicity and usefulness of the procedure and written consent was taken from relative or patient himself.

The patient is placed in the lateral decubitus position, with the top leg flexed and the lower leg straight. The site is prepared, cleaned with an antiseptic (usually Betadine) scrub, and draped, exposing only the biopsy area. The skin and the area down to the periosteum are infiltrated with a local anesthetic (eg, 1\% Xylocaine). Approximately $10 \mathrm{cc}$ of $1 \%$ Xylocaine is used. A skin incision is made with a small surgical blade, through which the bone marrow aspiration and biopsy needles are inserted. The bone marrow aspiration needle, with a stylet in place, is inserted. Once the needle contacts the bone, it is advanced by rotating clockwise and counterclockwise slowly until the cortical bone is penetrated and the marrow cavity is entered. Usually, a sudden change is noted when the marrow cavity has been entered. Once within the marrow cavity, the stylet is removed, and, using a 20 -cc syringe, approximately $0.5 \mathrm{cc}$ of bone marrow is aspirated for pathology slides. Slides are made and fixed it with methanol after drying. The marrow aspiration needle is removed, and pressure is applied to the site with gauze until bleeding has stopped. Following this procedure, a bone marrow biopsy usually is performed. The needle is held with the palm and index finger, and the stylet is locked in place. Once the needle touches the bone, the stylet is removed. Using firm pressure, slowly rotate the needle in an alternating clockwise-counterclockwise motion and advance it into the bone marrow cavity to obtain an adequate bone marrow specimen measuring approximately 1.5-2 $\mathrm{cm}$ in length. Rotate the needle along its axis to help cut the specimen, pull back approximately 2-3 $\mathrm{mm}$, and advance the needle again slightly, at a different angle, to help secure the specimen. Following this procedure, slowly pull the needle out while rotating in an alternating clockwise and counterclockwise motion. Remove the specimen from the needle with the probe supplied by introducing the probe through the distal cutting end. Place the specimen in formalin solution for histology processing.

After the procedure, several layers of gauze are applied to the site with an Elastoplast on top to immobilize the gauze, and the patient is instructed to check the site frequently, to report any bleeding, and to keep it dry. The dressing is removed 48 hours later.

Bone marrow aspiration slides are stained with Giemsa stain and special stains like Perl, Sudan, PAS whenever needed. Bone marrow biopsies were processed and stained with hematoxylin and eosin and special stains like PAS whenever needed.

OBSERVATION AND RESULTS: Total 27 bone marrow bone marrow trephine biopsy were performed. The number of bone marrow trephine biopsy performed is small because most the cases bone marrow aspiration was diagnostic like in cases of megaloblastic anemia, iron 
deficiency anemia. Trephine biopsy is really helpful when there is a dry tape. Table 1 shows distribution of cases in bone marrow biopsy. Table 2 shows comparative evaluation of bone marrow cellularity by BMA and BMB.

Table 2 shows that number of case of dry tape and heavy admixed with blood which cause difficulty in evaluation the marrow was quite high(nearly $30 \%$ case) which may be due to wrong method or fibrosis or packed marrow. Out of 5 dry tap 3 were hyper cellular marrow and failure of aspiration is due to packed marrow. In those cases trephine biopsy proved to be diagnostic, and it also gives advantage of smear cytology by imprints smears of marrow. On aspiration smears, assessment of cellularity is not ideal for that biopsy is turn out to superior.

DISCUSSION: BMA and BMB are important for diagnosis of many hematological and non hematological malignancies, assessment of pyrexia of unknown origin, typing of anemia and in some infections. They also help in follow up of patient taking chemotherapy, bone marrow transplantation. $[5,6,7,8]$

BMA used to study morphology, maturation stages of blood cells, differential counts, and assessment of myeloid to erythroid ratio. [3] While trephine biopsy gives information about cellularity, architecture and focal lesions like granuloma, metastasis.

As noted in table 2, there was a wide discrepancy between cellularity of the marrow as estimated by biopsy versus aspirate.

In hyper cellular marrow, bone marrow reported as hyper cellular on aspiration method was $54 \%$ which was similar to ralph [9] study. In present study remaining $46 \%$ cases was either dry tap or reported as heavy admixed with blood and not possible to assess the cellularity. In normo cellular marrow, marrow was reported as normo cellular on aspiration was only in $37.5 \%$, while remaining $62.5 \%$ cases reported as either hyper cellular or hypo cellular marrow. In hypo cellular marrow, only $16.5 \%$ marrow was reported as hypo cellular by aspiration.

An important limitation of marrow examination obtained by aspirate is the admixing of marrow and sinusoidal blood, which may not allow for reliable estimates of marrow cellularity. This evaluation is of particular importance in the hyper cellular marrow which yields a "dry tap" or only dilutes sinusoidal blood. Thus, the use of the biopsy avoids misinterpretation of cellularity by smears in patients in whom the biopsy confirmed a normal, hypo cellular or hyper cellular marrow.

In present study percentages of dry taps are $18 \%$, out of which $80 \%$ marrow are abnormal on biopsy. Only $20 \%$ marrows are normal. Table 3 shows comparative evaluation of dry tap in various studies. Out of 5 cases of dry tap one was acute leukemia, one was multiple myeloma, one was essential thrombocytosis. In these cases dry tap was due to pack marrow. One dry tap was due to myelofibrosis. One was normal marrow which may be due to faulty technique. It is necessary that finding of a 'dry tap' should never be dismissed as being due to faulty technique and always needs a bone marrow biopsy.

In this study, there were three case of Chronic Lymphoid Leukemia and all three have diffuse involvement of marrow, which can be seen on biopsy section, while aspiration only shows that marrow is involved. Pattern of marrow involvement by leukemic cells could be only analyzed by trephine biopsy. Not all patients with Chronic Lymphoid Leukemia required bone marrow examination because it is easily made by peripheral examination. However bone marrow assessment is indicated in patients in whom treatment is necessary, either those with more advanced disease or younger patients in whom intensive treatment is planned.[13] This should always include a trephine biopsy because bone marrow aspirate gives very little 
information beyond that already available from examination of blood, where as trephine biopsy permits an accurate assessment of extend of infiltration and gives information of prognostic importance ${ }^{[14]} \mathrm{A}$ trephine biopsy is essential for follow up of intensive treatment because it may show residual focal disease when a marrow aspirate is normal..[13]

In present study Reticulin stain was done and graded from 0 to 4 grades.

Out of 27 cases one case was of myelofibrosis [figure 3] with grade 4 Reticulin fiber, while those with leukemia had grade 1 to 2 Reticulin fiber contain. While those with anemia and normal marrow doesn't have any demonstrable reticulin fiber (grade 0 ).

In our study there are two cases of metastasis one from lymphoma and other from neuroblastoma[figure 4]. The combined procedures of aspiration and biopsy give a higher yield and are essential in patients with suspected carcinoma, non Hodgkin lymphoma and Hodgkin lymphoma.[15] Bilateral aspirates with biopsies are required for diagnosis and in staging of Neoplasms and that a unilateral aspirate with biopsy is sufficient to assess patients with cytopenia or leukemia.[16]

In present study aspiration alone was sufficient in making a diagnosis in $70 \%$ cases. In these cases trephine biopsy gives additional information. In the remaining $30 \%$ cases trephine biopsy was necessary for making a diagnosis due to incomplete information provided by aspiration or its inability to give a correct diagnosis.

The decision to perform a marrow aspiration alone or in combination with marrow biopsy depends on the diagnosis being considered. In nutritional anemias, most hematologic malignancies and immune thrombocytopenia, marrow aspiration alone is sufficient, but for detection of disorders with focal marrow involvement biopsies are must. [17]

CONCLUSION: Aspiration is easy to perform while trephine biopsy is slightly more painful and requires more skill to perform. Aspiration is important to study the cytology of bone marrow while biopsy is more reliable in assessing cellularity and reticulin framework of marrow. Aspiration is far superior in case of anemia, leukemia while trephine biopsy is more useful when aspiration yield dry tape or heavy admixed with blood. It also gives much more information regarding abnormal localization of immature cells, pattern of metastasis, post chemotherapy to evaluate how marrow is responding to treatment or even in bone marrow transplantation.

Overall both the procedures are complimentary to each other and must be performed together for better evaluation of patient.

\section{REFERENCES :}

1. Sabharwal BD, Malhotra V, Aruna S, Grewal R. Comparative evaluation of bone marrow aspirate particle smears, imprints and biopsy sections. J Postgrad Med 1990; 36(4):194-8.

2. Antonino Musolino, Annamaria Guazzi, Rita Nizzoli, Michele Panebianco, Cristina Mancini and Andrea Ardizzoni. Accuracy and relative value of bone marrow aspiration in the detection of lymphoid infiltration in non-Hodgkin lymphoma. Tumori 2010; 96:24-7.

3. Hamid GA, Hanbala N. Comparison of bone marrow aspiration and bone marrow biopsy in neoplastic diseases. gulf j oncology 2009 Jul;(6):41-4.

4. M Naznin[a], A J Wahab[b], R Kalavathy[c].A review of bone marrow examinations in tengku ampuan afzan hospital (htaa), kuantan. 
5. Lee SH, Erber WN, Porwit A, Tomonaga M, Peterson LC; International Council for Standardization In Hematology.ICSH guidelines for the standardization of bone marrow specimens and reports. . Int J Lab Hematol. 2008 Oct;30(5):349-64.

6. Pampa Ch Toi, Renu G'Boy Varghese, Ramji Rai. Comparative Evaluation of Simultaneous Bone Marrow Aspiration and Bone Marrow Biopsy: An Institutional Experience. Indian J Hematol Blood Transfus.2010; 26(2):41-4.

7. Riley RS, Hogan TF, Pavot DR, Forysthe R, Massey D, Smith E, Wright L Jr, Ben-Ezra JM.A pathologist's perspective on bone marrow aspiration and biopsy; Performing a bone marrow examination. J Clin Lab Anal 2004; 18(2):70-90.

8. Islam A. Bone marrow aspiration prior to bone marrow core biopsy using the same bone marrow biopsy needle. A good or bad practice. J Clin Pathol. 2007; 60(2):212-5.

9. Gruppo RA, Lampkin BC, Granger S. Bone marrow cellularity Determination: Comparison of the Biopsy, Aspirate, and buffy coat. Blood 1977; 49(1):29-31.

10. Navone R, Colombano MT. Histopathological trephine biopsy findings in cases of 'dry tap' bone marrow aspirations. Appl Pathol 1984; 2(5): 264-71.

11. Engeset A, Nesheim A, Sokolowski J. Incidence of 'dry tap' on bone marrow aspirations in lymphomas and carcinomas.Diagnostic value of the small material in the needle. Scand J Haematol 1979; 22(5), 417-22.

12. Humphries JE. Dry tap bone marrow aspiration: clinical significance. Am J Hematol. 1990; 35(4):247-50.

13. B J Bain. Bone marrow trephine biopsy. J Clin Pathol.2001; 54:737-42.

14. B J Bain. Bone marrow aspiration J Clin Pathol 2001; 54:657-663.

15. James D. Bearden, Gary A. Ratkin, and Charles A. Coltman. Comparison of the diagnostic value of bone marrow biopsy and bone marrow aspiration in neoplastic disease. J clin. Pathol. 1974;27(9): 738-740.

16. Barekman CL, Fair KP, Cotelingam JD. Comparative utility of diagnostic bone-marrow components: a 10-year study. Am J Hematol. 1997;56(1):37-41.

17. Annu Nanda, Sabita Basu, Neelam Marwaha. Bone Marrow Trephine Biopsy as an Adjunct to Bone Marrow Aspiration. J Assoc Physicians India 2002;50:893-5.

\section{TABLE 1: Distribution of Cases.}

\begin{tabular}{|l|l|l|l|l|l|}
\hline $\begin{array}{l}\text { Type of } \\
\text { Disease }\end{array}$ & Normal & Anemia & $\begin{array}{l}\text { Acute } \\
\text { Leukemia } \\
\text { [figure 1] }\end{array}$ & $\begin{array}{l}\text { Chronic } \\
\text { lymphoid } \\
\text { Leukemia }\end{array}$ & Metastasis \\
\hline No. & 07 & 04 & 04 & 03 & 02 \\
\hline $\begin{array}{l}\text { Type of } \\
\text { Disease }\end{array}$ & $\begin{array}{l}\text { Myelo } \\
\text { fibrosis }\end{array}$ & $\begin{array}{l}\text { Chronic } \\
\text { Myelo } \\
\text { Proliferative } \\
\text { Disorders }\end{array}$ & Myeloma & $\begin{array}{l}\text { Storage } \\
\text { Disease } \\
\text { [Figure 2] }\end{array}$ & Total \\
\hline No. & 01 & 04 & 01 & 01 & $\mathbf{2 7}$ \\
\hline
\end{tabular}


Table 2. Comparative evaluation of bone marrow cellularity by BMA and BMB.

\begin{tabular}{|l|l|l|l|l|}
\hline & & BMB & & \\
\hline & hypocellular & normocellular & hypercellular & Total \\
\hline BMA & & & & \\
\hline Hypocellular & 01 & 01 & 00 & 02 \\
\hline Normocellular & 02 & 03 & 00 & 05 \\
\hline Hypercellular & 02 & 03 & 07 & 12 \\
\hline Dry tap & 01 & 01 & 03 & 05 \\
\hline Blood mixed & 00 & 00 & 03 & 03 \\
\hline & 06 & 08 & 13 & 27 \\
\hline
\end{tabular}

Table 3: comparison of dry tap in various studies

\begin{tabular}{|l|l|l|l|}
\hline Various study & $\begin{array}{l}\text { Dry tap in } \\
\text { aspiration[\%] }\end{array}$ & $\begin{array}{l}\text { Normal marrow in } \\
\text { biopsy[\%] }\end{array}$ & $\begin{array}{l}\text { Abnormal marrow in } \\
\text { biopsy[\%] }\end{array}$ \\
\hline Navone R et al[10] & 5 & 0 & 100 \\
\hline Engeset A et al[11] $^{[12]}$ & 7 & 23 & 77 \\
\hline Humphries JE[12] & 4 & 7 & 93 \\
\hline Present study & 18 & 20 & 80 \\
\hline
\end{tabular}

\section{ACUTE LEUKEMIA}

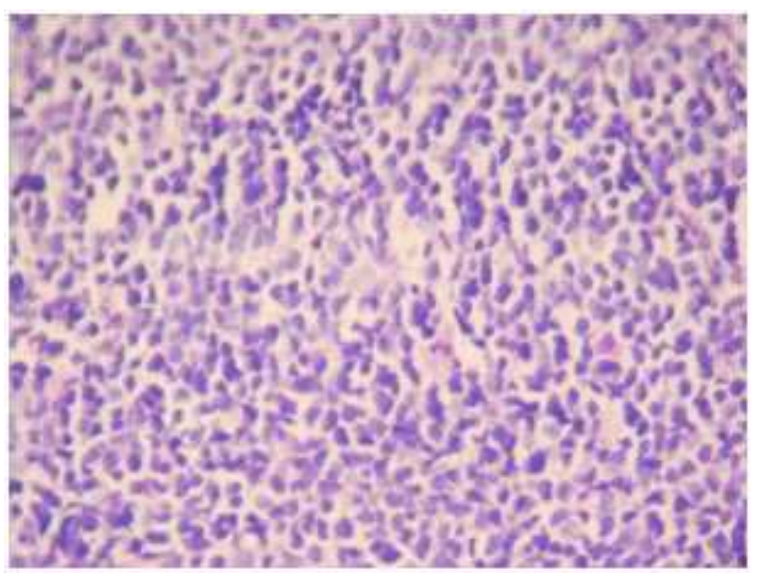

Figure 1.Acute leukemia - BMB shows blasts infiltrating the marrow and almost replacing the normal marrow [h \& e stain 400x] 


\section{GAUCHER'S DISEASE}

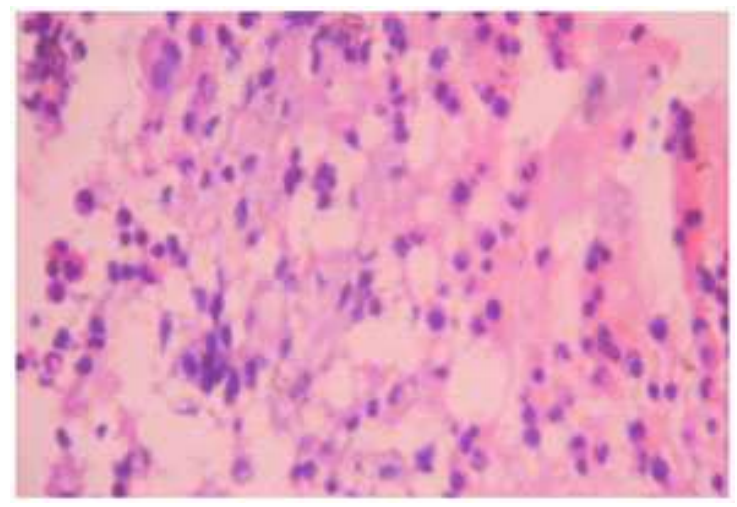

Figure 2. Gaucher's disease - BMB shows many gaucher cell infiltrating marrow and they are PAS positive [PAS stain 400x]

MYELOFIBROSIS:

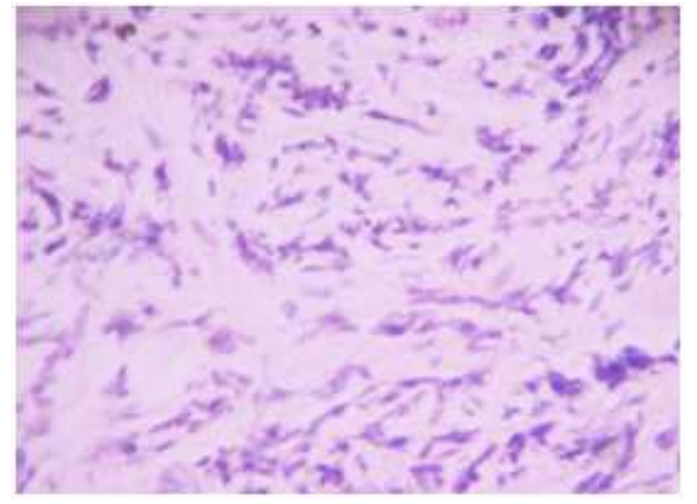

Figure 3.Myelofibrosis - BMB shows increased fibrosis and collagen deposition in myelofibrotic marrow [ $h$ \& e stain 400x ]

\section{NEUROBLASTOMA:}

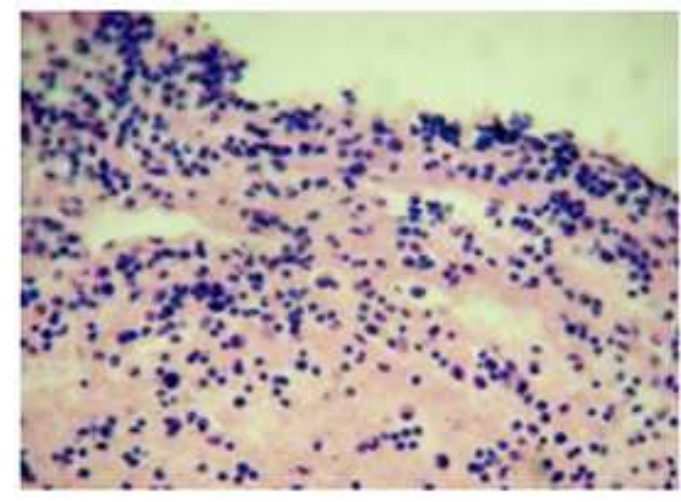

Figure 4.Metastatic neuroblastoma - section shows small round cells with scant cytoplasm infiltrating the marrow and fibrillary background 